\title{
La dimension orphique de la musique mongole
}

\section{Alain Desjacques}

\section{OpenEdition}

\section{Journals}

Édition électronique

URL : http://journals.openedition.org/ethnomusicologie/2384

ISSN : 2235-7688

Éditeur

ADEM - Ateliers d'ethnomusicologie

Édition imprimée

Date de publication : 1 janvier 1990

Pagination : $97-107$

ISBN : 2-8257-0423-7

ISSN : $1662-372 X$

Référence électronique

Alain Desjacques, "La dimension orphique de la musique mongole », Cahiers d'ethnomusicologie [En ligne], 3 | 1990, mis en ligne le 15 octobre 2011, consulté le 19 avril 2019. URL : http:// journals.openedition.org/ethnomusicologie/2384 


\title{
LA DIMENSION ORPHIQUE DE LA MUSIQUE MONGOLE
}

\author{
Alain Desjacques
}

\begin{abstract}
"Dans la vie ordinaire parmi les gens pacifiques, conduisez-vous comme des veaux âgés de deux ans, dans les querelles attaquez comme des vautours; pendant les festins et les divertissements soyez comme de jeunes étalons, mais dans le combat avec les ennemis attaquez, foncez comme des faucons affamés sur leur proie; ... par une journée claire soyez vigilants comme un loup endurci; par une nuit sombre, prudents comme le corbeau..."
\end{abstract}

Genghis Xan

En mongol, musique se dit xögžim, dérivation nominale de xög («ton» ou «mélodie») ${ }^{1}$, sur lequel a été formé le mot xögžil («développement»). Le terme $x \ddot{g} g z i m$, se trouvant dans un voisinage linguistique étroit avec xögžil, pourrait faire croire que la musique est perçue, chez les Mongols, comme une durée, le processus temporel d'un événement sonore. En réalité, les musiciens populaires entretiennent un rapport plus concret avec le milieu naturel et géographique dans l'élaboration de leurs genres musicaux, ainsi que l'illustre cette remarque, teintée de poésie, de S. Süren:

«La longueur et la lenteur de nos chants longs (urtyn duu) traduisent l'immensité de la steppe et les grands intervalles ont été inspirés par la hauteur et la configuration des sommets de l'Altaï» ${ }^{2}$.

1 En kazakh, küü vient du chinois ch'ü, et du moyen chinois $k$ 'iok, «chanson», ajoute Rémy Dor qui a bien voulu relire ce texte. Que soient également remerciés les professeurs Ernst Emsheimer, Tràn Van Khê, Denise Hemmerdinger et Roberte Hamayon pour leurs remarques pertinentes.

3 D'après un entretien avec Sodovyn Süren, 74 ans, Mongol Urjanxaj de la province de Duut dans la région de Khovd, le 21 octobre 1984. 

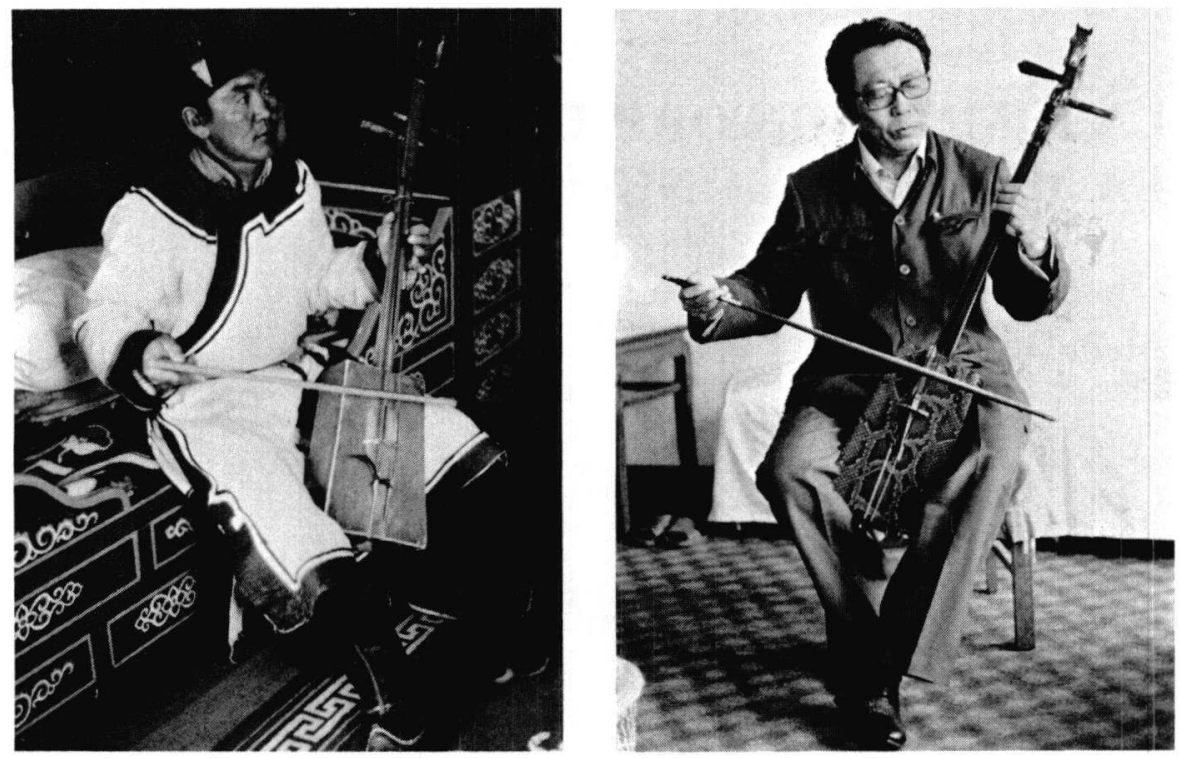

Fig. 1 et 2: La morin-xuur (vièle-cheval) est devenue l'instrument national de la République populaire de Mongolie. La caisse de l'instrument, de forme trapézoïdale, est traditionnellement recouverte d'une peau de chèvre souvent richement décorée. La morin-xuur tenue entre les jambes en position assise, accompagne principalement le «chant long» urtyn duu dans ses évolutions mélodiques, et les mouvements de la danse bijelge. Photos: Alain Desjacques.

Ce parti pris de «longueur et de lenteur» caractérise d'ailleurs bien ce type de chant dont la pulsation rythmique apparaît largement subordonnée au développement de la mélodie dont l'improvisation ornementale, soutenue par des techniques vocales particulières, donne l'impression d'un étirement mélodique du chant presque sur chaque syllabe.

Ce rapport quasi immédiat à l'environnement quotidien du pasteur nomade transparaît aussi clairement à travers le répertoire instrumental populaire qui fait une place importante à l'imitation des bruits de la nature, du chant des oiseaux et du comportement des animaux observés. Il est probable que, dans des temps très reculés, il s'agissait surtout de reproductions de cris d'animaux lors d'activités spécifiques, telle la chasse, où l'appeau devait être un instrument des plus fidèles. Il serait tentant de penser que l'imitation est, sinon postérieure à la reproduction sonore, du moins une forme artistique de celle-ci. Ces imitations ne sont, bien sûr, pas exemptes de fonctions sociales et religieuses, comme dans les rites de chasse ${ }^{3}$ ou bien dans les séances chamaniques

3 Notamment les rites de chasse à l'ours et de consommation de sa chair dans un banquet durant lequel, chez les Yakoutes et les Toungouzes, on imitait le croassement du corbeau pour faire croire à l'ours que ce ne sont pas des hommes mais des oiseaux qui sont en train de le manger (cf. Harva 1959: 296-97). 
durant lesquelles l'habileté du chamane à l'imitation, pas seulement sonore d'ailleurs, constitue un élément indéniable de son art. Et Dodds (1959: 151) écrit à propos d'Orphée:

«Comme certains chamanes légendaires de Sibérie, il peut par sa musique convoquer les bêtes et les oiseaux pour se faire entendre».

Il se pourrait bien que l'essence de la musique instrumentale populaire mongole soit une musique de la nature, une musique animalière en rapport avec le système religieux du chamanisme. L'origine légendaire de la morinxuur et de son répertoire offre, de ce point de vue, un apport très intéressant ${ }^{4}$.

Dès le début de la légende, le héros-chanteur reçoit le qualificatif de xöxöö qui désigne le coucou en mongol. En effet, aujourd'hui encore en Mongolie, il n'est pas rare d'attribuer le surnom honorifique de xöxöö à un excellent chanteur ou à une excellente chanteuse, soulignant par là le rapport entre la perfection de son art et le chant d'un oiseau. Ce dicton mongol est souvent utilisé pour qualifier un chant particulièrement beau:

\section{Nuurand öndöglösöm \\ «C'est le chant d'une cane \\ Nugasny duu gargaad \\ Qui a pondu au bord du lac, \\ Xulsan öndöglösön \\ Xungijn duu gargaad. \\ C'est celui du cygne \\ Qui a pondu dans les roseaux.»}

Mais surtout, l'onomatopée xöxöö désignant le coucou ne résulte pas d'un choix linguistique arbitraire. Il semble en effet que, par similitude phonique, les Mongols aient rapproché le chant du coucou du mot xöx («bleu»), dénomination du «ciel» en mongol, ce qui confère au coucou un rôle très important dans la mythologie mongole, car, comme nous le verrons, ce n'est pas seulement un oiseau mais aussi un messager du ciel.

Le mot $x \ddot{x} x$ désigne le ciel, c'est-à-dire le dieu du ciel: «Ce qui est curieux, c'est que les Mongols aient pu, dans leur adoration, appeler le dieu du ciel "Ciel Bleu” ", s'étonne Harva (1959: 103) qui donne plus loin cette information:

«Dans un dessin représentant la montée du chamane dans les cieux, nous voyons, à côté du dieu suprême et de "son messager", les figurations de trois autres êtres célestes. Selon une explication jointe à ce dessin et qui provient probablement des šors, on y reconnaît Bogdigan et aussi Bobyrgan, un être légendaire; la troisième figure représente le kökyš» (1959: 112).

4 La version la plus répandue de la légende sur l'origine de la vièle morin-xuur a été récemment éditée par Sampildendev et Cerensodnom (1984, légende $\left.\mathrm{N}^{\circ} 79\right)$. Elle commence ainsi: «Il y a très longtemps vivait à l'extrême Est de la Mongolie un homme brave nommé Xöxöö Namžil, chanteur de grande réputation au niveau régional. Mais il fut mobilisé par l'armée qui l'envoya à l'Ouest» (c'est-à-dire dans le massif de l'Altaï). 
Malheureusement, Harva ne s'étend pas davantage sur le kökys. Nous pensons que c'est le coucou. En effet, Ulgen, c'est le «bon» dieu; son antagoniste se nomme Erlig, dieu du monde souterrain. Chacun de ces dieux est entouré d' «assistants» et de «fils» qui interviennent dans la lutte divine. Que $k \ddot{k} k y \breve{s}$ soit un allié de Erlig ne serait pas étonnant. Le coucou est un oiseau de mauvais augure.

«Le corbeau, la corneille ${ }^{5}$ et le coucou sont des oiseaux omineux [sic]», et: «pour les Yakoutes, la mort est annoncée par le coucou» (Roux 1966: 391, 73). C'est donc un oiseau extrêmement dangereux que le chamane doit vaincre dans sa montée céleste, et Harva le confirme en citant un manuscrit dans lequel «un chaman au début de la séance invoque l'aide d'un fils d'Ulgen nommé Karšit». C'est sans doute un oiseau de proie et, peut-être plus précisément, karšit est une variante linguistique de xař̌yga en kazakh et xarcaga ou xarcagaj en mongol pour l'autour des palombes (accipiter gentilis). En effet, l'oiseau de proie dressé peut se révéler redoutable pour le coucou, comme le montre la suite du manuscrit où «un oiseau noir», Karakus ( «aigle»), est un serviteur du chamane: «on raconte que le chamane, à son arrivée dans le quatrième ciel, montre comment Karakus prend un coucou» (Harva 1959: 113). Le chamane essaiera d'ailleurs d'accaparer le pouvoir du coucou: «les os du coucou sont employés pour faire des charmes et des talismans» (Roux 1966: 164).

Mais la suite de la légende sur la morin-xuur indique que Xöxöö Namžil fut mobilisé par l'armée qui l'envoya à la frontière ouest de la Mongolie et que son chef, dès qu'il eut connaissance de la beauté de son art, lui épargna l'entraînement militaire pour lui permettre de se consacrer entièrement à l'art du chant. Il est donc exempt de tâches viriles. Est-il considéré comme une femme? Est-il une femme? Un mythe d'origine du coucou recueilli chez les Tatars de l'Altaï affirme que «les coucous et les hérons ne seraient que des jeunes filles méconnaissables»; chez les Toungouzes, «une jeune fille se change en coucou par désir de boire» (Roux 1966: 35, 246).

Pendant le temps de son service à l'armée, il fit la connaissance d'une très belle princesse qui lui fit cadeau d'un cheval magique appelé Žonon Xar. Il était représenté ainsi:

«Tandis que par sa vitesse il détache les broussailles de leurs racines,

Qu'il brise les rochers en morceaux,

Qu'il disperse les amas de pierres,

Qu'il déchire la couture du vêtement du cavalier.

Il ne glisse pas sur le roc,

Il trotte sans trébucher dans les fourrés,

Lorsque l'âme de Tarvaa, le barde légendaire de Mongolie, revient de chez Erlig (dieu du monde souterrain), elle s'aperçut que les yeux de son corps laissé sur place avaient été picorés par une corneille, causant sa cécité (sur cette légende et le rapprochement de Tarvaa avec Homère, voir Doulam 1987: 36-7). 
Il n'a rien à envier aux oiseaux ${ }^{6}$,

Il n'est pas comparable à un cheval ordinaire,

Dans le troupeau il est l'unique,

Il a tout à fait l'allure d'un cheval de course,

Au moment du danger il vole en chantant,

Au moment du repos il va d'une démarche trottinante,

Quand son maître le chevauche

Il se donne de la peine,

C'est l'ami de l'homme,

C'est un bon cheval.»

Un tel cheval se rencontre aussi dans les contes ou les chants épiques mongols (cf. Doulam 1987). Cependant, le cheval occupe une place importante dans la mythologie altaïque qui retient le thème du cheval ailé pour justifier l'origine mythique du cheval. Le cheval ailé est aussi une des montures du chamane, car:

«[...] l'homme ne peut s'élever dans les airs, s'il ne voyait accourir à son aide celui qui sait voler: l'oiseau d'abord ou, à défaut, un quelconque animal qui n'a pas oublié comment on acquérait des ailes. C'est le thème de Pégase que l'on rencontre souvent» (Roux 1966: 65).

Mais surtout, le cheval a été souvent identifié au soleil. Dans le système astrologique du calendrier chinois des douze animaux, emprunté par les Mongols, le mois de juin, c'est-à-dire celui du solstice d'été, est le mois du cheval; et midi, le moment où le soleil atteint le zénith dans sa course quotidienne, s'appelle l'heure du cheval ${ }^{7}$. Rien d'étonnant alors que le cheval soit «un animal essentiel dans les cérémonies de sacrifices au ciel» (Roux 1966: 78). Pour nous, il apparaît bien que le cheval, ainsi que le coucou qui se trouve sur la route du chamane dans sa montée céleste, sont deux aspects zoomorphes différents d'une même réalité, le soleil, qui fait penser au mythe d'Icare. La suite de la légende montre Xöxöö Namžil chevauchant son cheval Žonon Xar, la nuit tombée, pour aller retrouver sa princesse à l'Ouest du pays et rentrant à l'aube à l'Est. La légende fait donc coïncider le retour du cheval avec l'apparition du soleil à l'est.

Mais, dans cette légende, une femme jalouse du campement ${ }^{8}$ découvrit le secret et coupa les ailes du cheval qui mourut sur le champ. Lorsque Xöxöö Namžil constata plus tard la mort de son cheval, il sombra dans une profonde

6 C'est-à-dire qu'il est ailé.

7 En kazakh, kök désigne à la fois la couleur bleue et le ciel, kökek le coucou et le mois de juin.

8 Dans une autre version, il s'agit de la propre femme du héros (cf. la légende recueillie par Haslund-Christensen in Emsheimer 1943: 35-7). 
tristesse. C'est alors qu'il se mit à sculpter la tête de son cheval dans un long morceau de bois qu'il fixa sur un bol en bois; il le recouvrit de la peau de son cheval. De sa belle queue soyeuse, il prit du crin qu'il tendit sur l'instrument et qu'il enduisit de brai sec. Xöxöö Namžil imita ainsi les hennissement de Žonon Xar, son allure et les différents aspects de sa démarche. Une autre version de la légende dit que c'est le crâne même du cheval qui servit de caisse de résonance ${ }^{9}$. Ainsi, dans la légende, un instrument est créé, la morin-xuur, pour un répertoire spécifique, constitué d'imitations du hennissement du cheval, de sa démarche, et d'interprétations sonores de son comportement et de son caractère. Aujourd'hui encore, de telles imitations apparaissent dans le jeu instrumental du musicien xuurč qui chante en l'honneur d'un cheval, comme dans la «Louange au beau cheval brun», l'«Eloge de l'alezan à l'étoile frontale blanche, originaire du pays des Khotgojd», mais aussi dans la musique de danse avec, par exemple, "Le coursier noir", "Le cheval ambleur», "Le bruit des sabots du cheval", "Le cheval noir de trois ans", et "La démarche du cheval ${ }^{10}$. Mais la légende ne donne pas d'explication sur l'origine du mot xuur, de l'expression morin-xuur (littéralement «vièle-cheval»). Pour E. Haenish (1939: 73), le terme de xuur, en mongol ancien qugur,

«[...] désigne toujours un instrument à cordes pincées ou a cordes frottées. D'un point de vue phonétique, il semble dériver du turc ancien qopuz avec différentes variantes dans les langues de nombreux groupes ethniques, qui signifie “instrument à cordes". »

Il devient alors plus difficile de comprendre pourquoi les Mongols utilisent le même terme pour désigner la guimbarde aman-xuur, avec les variantes xulsan xuur si elle est en bambou et tömör xuur si elle est en fer ${ }^{11}$. Or, la traduction par «vièle à bouche» n'est pas satisfaisante. D'ailleurs, comme pour dissiper un malentendu, les Mongols emploient parallèlement le terme de tobšuur, qui désigne le luth à deux cordes à l'Ouest de la Mongolie, pour la guimbarde. Et, en effet, pour jouer de la guimbarde, il faut pincer (tobšix) la languette, comme il faut pincer les cordes du luth. En fait, qugur ou qagur en mongol ancien veut dire originellement $\left\langle\right.$ sifflement du vent ${ }^{12}$, devenu avec l'introduction de l'alphabet cyrillique xuur ou xuguur, avec une dérivation verbale xuugix: «siffler» (pour le vent). Le rapport entre le sifflement du vent, le frot-

D'après une information de Doržbalam, 66 ans, peintre émérite, originaire du sud de la Mongolie, région de Dundgov, septembre 1986.

10 Les titres mongols de ces morceaux, respectivement: Xöörxön zeerd moriny magtaal, Xotgojdyn ündesnij xongor xalsan mor' magtaal, Žalam xar, Demen xar, Morin tövörgöön, Gunan xar, et Moriny javdal.

11 Temür komus en kazakh.

12 D'après une information de Amsgalan, peintre émérite d'origine zaaxčin de l'ouest de la Mongolie, septembre 1986. 


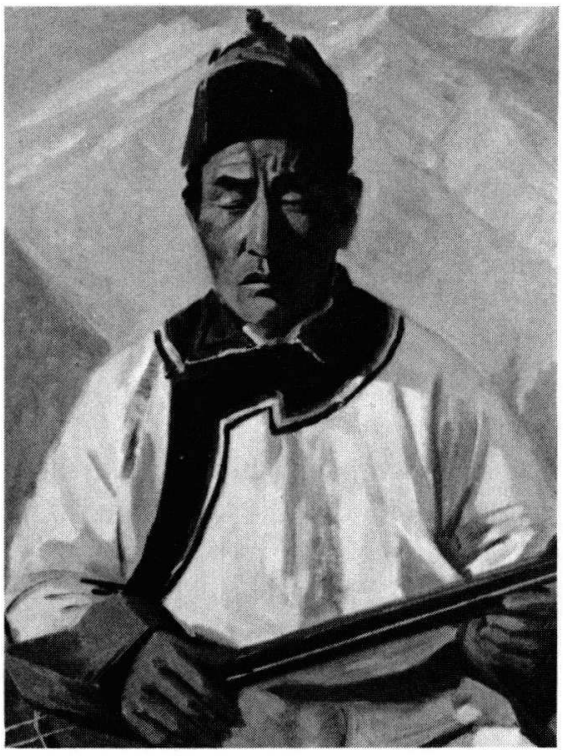

Fig. 3: Le barde Avirmed, au visage expressif d'une étonnante concentration intérieure, chante encore l'épopée tuul' dans un style guttural appelé xajlax, «forger l'épopée». Il s'accompagne d'un luth à deux cordes tobšuur, typique de la région de l'Altaï. Peinture de S. Bold, 1986. Photo: Alain Desjacques.

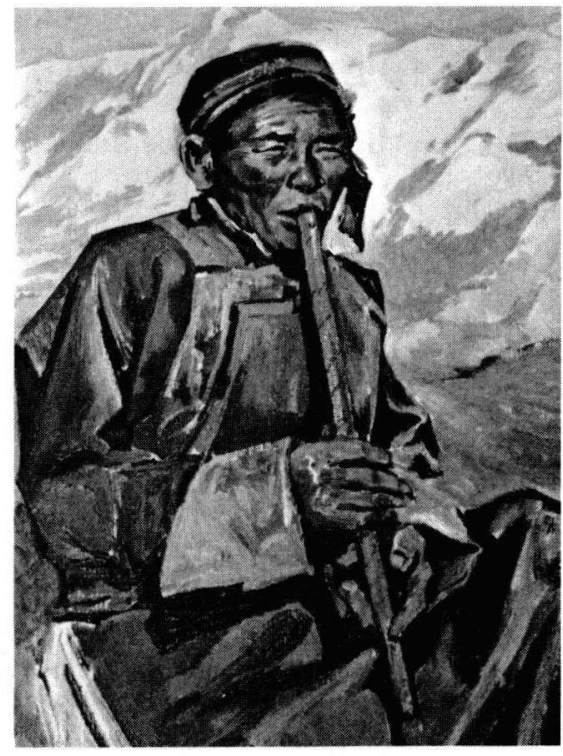

Fig. 4: Le ruissellement d'une source montagneuse, le chant d'un oiseau et le subtil murmure de la nature incitent le berger à leur répondre au son de sa flûte cuur. P. Narangogt, peint par S. Elbegdorz, 1986. Photo: Alain Desjacques.

tement de l'archet sur les cordes et le son de la guimbarde devient alors plus évident. Une onomatopée mongole traduit le frottement de l'archet par ilme žlme ${ }^{13}$, c'est-à-dire par un chuintement, avec la fricative $\breve{z}$, qui est un élément descriptif possible du son de la guimbarde et du bruit du vent, car il semblerait bien que, pour les Mongols, le vent chuinte, alors que pour nous, il siffle.

D'autre part, chez les peuples altaïques, le chuintement du vent est associé à la montagne:

«Les Iakoutes disent que les vents "dorment" sur la montagne d'où on peut les appeler en cas de besoin et en sifflant. Les Golds croient que les vents sortent des gorges montagneuses où ils sont conservés par l'esprit du vent. Les Mongols appellent le temps des tempêtes "jours de course", dans la croyance que les esprits de la montagne courent alors d'une montagne à l'autre.» (Harva 1959: 158).

13 «C'est au son de ilme žlme que tu frottes les cordes de la vièle ekil» : couplet d'un chant intitulé «Tu m'as dit que...», recueilli par Doulam (1987: 45). 
Et récemment, nous avons appris auprès de P. Narantsogt, Mongol Urjanxaj de l'Altaï, que:

«[...] la mélodie fondamentale (xiigin ajalguu) de la flûte verticale cuur, instrument de berger, provient certainement de quelqu'un qui, se trouvant seul sur une montagne, regarda le paysage à l'écoute du chuintement du vent et eut l'idée de lui répondre comme un "chant diphonique" des montagnes. " ${ }^{14}$.

Dans la légende précédente, le héros Xöxöö Namžil est allé à l'Ouest de la Mongolie, c'est-à-dire dans la région montagneuse de l'Altaï, puis est retourné dans son pays natal à l'Est où il créa alors la morin-xuur.

Le chuintement du vent ${ }^{15}$, mais aussi le bruissement des herbes hautes, le clapotis de la rivière, le chant des oiseaux et les cris des animaux sont autant de sources d'inspiration pour le chasseur ou le berger des régions montagneuses. Le répertoire de Narantsogt est à ce titre éloquent. A l'aide de son pouce ou de son index, ou encore d'une simple petite pierre plate ramassée par terre et placée entre les dents, Narantsogt est capable d'imiter le coucou adulte, le jeune coucou, deux coucous qui se répondent, la huppe ordinaire, le pigeon ramier, le grand coq de Bruyère, et avec sa flûte cuur, le tétras-lyre, parmi beaucoup d'autres, imitant aussi bien les hommes que les animaux. Bien sûr, ces imitations sont actuellement dépouillées de toute connotation chamanique. Cependant, on ne peut nier la place importante du coucou dans la mythologie des Mongols et d'autres peuples altaïques. Mais quel est l'importance de ces imitations dans la musique mongole? Et quels sont les aspects retenus de la source sonore originelle? En effet, il ne s'agit presque jamais de reproduction exacte du chant des oiseaux ou des bruits de la nature, mais plus généralement d'une imitation qui se fonde sur un ou plusieurs traits caractéristiques de la source sonore. Dans le chant du coucou, par exemple, ce qui est important pour Narantsogt, c'est le bitonisme vocalisé du chant de cet oiseau qui va être interprété vocalement à l'aide d'une petite pierre plate dans l'intervalle d'une tierce mineure $\mathrm{sol}_{4}-\mathrm{mi}_{4}\left(\right.$ ou si ${ }_{4}$-sol\# 4 ), en commençant par le degré supérieur ${ }^{16}$ avec des varia-

14 Information recueillie par Doulam (1987: 43). Mais la révision de mon texte traduit a entraîné une correction erronée de «chant diphonique» par «écho». Pour P. Narantsogt, cette métaphore de «chant diphonique des montagnes» (uulyn xöömij) est une illustration de ce qu'il pense être une diphonie simultanée, constituée par le jeu mélodique de la flûte cuur et le sifflement ininterrompu du vent sur les sommets montagneux.

15 Le monde animal n'est d'ailleurs pas insensible au sifflement du vent: «Le cygne [...] rompt le silence quand il entend Apollon toucher la lyre, et le rossignol est attiré hors des grottes vers le soleil quand le zéphir fait entendre son chant» (Photius, codex 114, édité par Henri 1971).

Un coucou d'Afrique commence par le degré inférieur, nous communique Claude Chapuis, ornithologue, qui ajoute que les exemples d'oiseaux rapportés ici ont un registre de vocalisation grave (entretien du 19 janvier 1987). Le musicologue mongol Ž. Badraa affirme que l'émission vocale diphonique xöömii ( "pharynx») vient probablement de l'imitation du cri de la grue noire xarkhiraa, à cause des sons gutturaux (1986: 18). Kargyraa est aussi le nom donné par les Touvins à un type précis d'émission vocale utilisant un registre particulièrement grave. 
tions dans la tonalité et le rythme. Le chant du coucou n'est d'ailleurs pas toujours le même. Dans l'imitation du pigeon ramier et de la huppe ordinaire, c'est le type particulier d'émission vocale «consonantique» de ces oiseaux qui est retenu. Ces courtes imitations sont l'objet de petites œuvres musicales, tel «Le chant du tétras-lyre», joué à la flûte cuur avec bourdon vocal.

Mais la simple observation d'un animal, de sa démarche et de son comportement, peut inspirer des imitations musicales. Il serait trop long d'énumérer les morceaux se référant au cheval, dont la démarche et les allures ont largement contribué à composer la palette de rythmes utilisés, non seulement en Mongolie, mais dans tout l'univers du pastoralisme nomade d'Asie centrale. Entre autres thèmes rythmiques de cette région, celui de la démarche boiteuse de l'animal sauvage blessé par le chasseur, ou de l'animal domestique entravé par l'éleveur, est très important dans la formation des rythmes $a k s a k^{17}$ de cette musique de l'Altaï.

En résumé, nous avons vu que l'imitation sonore n'était pas gratuite mais faisait partie autrefois de tout un ensemble de représentations chamaniques. Aujourd'hui, l'imitation sonore ne semble conserver que son côté ludique, et elle est pensée comme telle par les musiciens mongols. L'imitation n'est jamais une reproduction parfaite de la source sonore, mais une interprétation quelquefois très fine de celle-ci. Le musicien va retenir, selon les cas, l'aspect mélodique ou tonal, ou bien un vocalisme, ou encore un rythme, dans son élaboration d'une imitation d'un son. Mais l'imitation peut aussi s'inspirer d'une observation visuelle d'un mouvement ou d'une forme statique du relief; le tableau suivant permet de visualiser les différents aspects théoriques de l'imitation sonore:

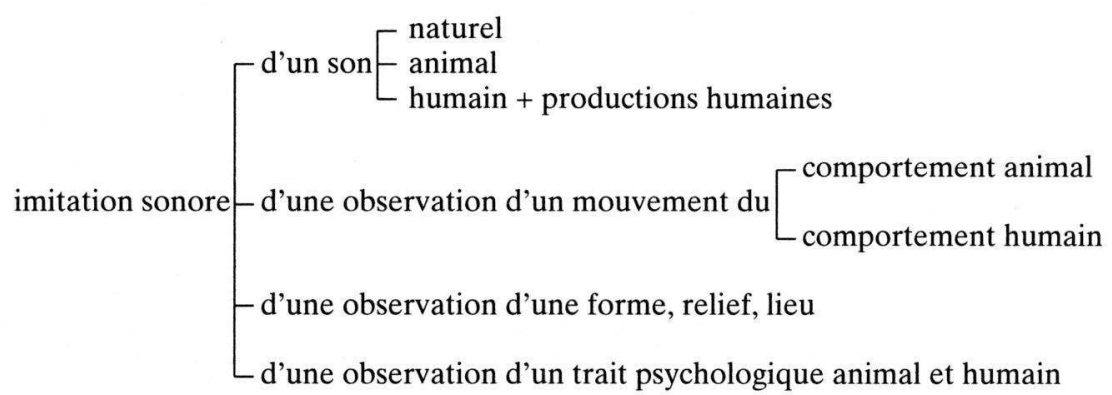

Ainsi plusieurs combinaisons sont-elles possibles.

17 Aksak veut dire «boiteux» en turc et en kazakh. Ce type de rythme fut analysé surtout par Constantin Brăiloiu, qui en a dressé un tableau complet (1973). 
C'est sans doute aussi sous cet aspect du rapport entre musique et environnement naturel et animal, qu'il est possible de penser l'originalité des émissions vocales diphoniques des Mongols, ou de l'usage abondant des pulsations glottales exécutées surtout dans les «chants longs» dont les degrés, bien qu'ils mettent en œuvre une échelle pentatonique constante, ne sont pas toujours bien stabilisés. Non pas parce que le chanteur chante «faux», mais parce que ce type de chant a aussi un rapport direct avec les appels du berger dans le milieu naturel du pasteur nomade (cf. Smirnov 1969: 238).

Il ne s'agissait pas ici d'aborder l'origine de la musique mongole, mais d'essayer de mettre en valeur un aspect particulier de cette musique caractéristique d'Asie centrale, qui conserve son visage singulier.

\section{Bibliographie}

AUBERT Laurent

1986 «Regards sur les musiques d'Asie centrale: La vièle-cheval et le luth-singe». Bulletin annuel du Musée d'ethnographie de la Ville de Genève 26: 27-51.

BADRAA $\breve{Z}$.

1986 «L'art xöömii». Les Nouvelles de Mongolie 9: 18-19.

BRAILOIU Constantin

1973 «Le rythme aksak» [1951]. In: Problèmes d'ethnomusicologie. Genève: Minkoff Reprints : 301-340.

DODDS Eric Robertson

1977 Les Grecs et l'irrationnel [1951]. Paris: Flammarion [Coll. «Champ philosophique», $N^{\circ} 28$ ].

DOULAM Sendenžavyn

1987 «Conte, chant et instruments de musique - quelques légendes d'origine mongole -. Etudes mongoles et sibériennes 18: 33-47. (Traduit du mongol par A. Desjacques, revu et corrigé par M.L. Beffa et F. Aubin).

\section{EMSHEIMER Ernst}

1943 «Preliminary remarks on Mongolian Music and instruments». In: The Music of the Mongols, Part I: Eastern Mongolia: 69-100. Stockholm: Tryekeri Aktiebolaget Thule. (Report from the Scientific Expedition to the Northwestern Provinces of China under the leadership of Dc Sven Hedin. The Sino-Swedish Expedition, Publication 21-VIII: Etnography, vol. 4)

1964 «Singing Contests in Central Asia». Studia Ethnomusicologica Eurasiatica (Stockholm) : $86-90$.

HAENISCH Erich

1935-39 Wörterbuch zu Manghal un niuca tobca'an, Die Geheime Geschichte der Mongolen. Teil 1: «Text aus der chinesischen Transkription im mongolischen Wortlaut wiederhergestellt». Teil 2: «Wörterbuch dazu». Leipzig.

HARVA Uno

1959 Les représentations religieuses des peuples altaïques. Traduit de l'allemand par J.L. Perret. Paris: Gallimard (Coll. «L'espèce humaine», $\left.N^{\circ} 15\right)$. 


\section{HEMMERDINGER Denise J.}

1982 «Atomisme et pythagorisme phéniciens aux origines des théories et des notations de la musique». In: Le livre et le Liban jusqu'à 1900. Paris: UNESCO, p. 47-57.

1984 «Aspects méconnus des théories et notations antiques et de leur transmission». In: Musicologie médiévale. Notations et séquences. Table ronde de paléographie musicale, Orléans, CNRS-IRHT, 6-7 septembre 1982. Paris: Editions du CNRS, p. 67-99.

HENRI R.

1971 La Bibliothèque de Photius, tome VI, codex 243. Paris: Éd. Les Belles Lettres.

LOT-FALCK Eveline

1953 Les rites de chasse chez les peuples sibériens. Paris: Gallimard (Coll. «L'espèce humaine», $\left.\mathrm{N}^{\circ} 9\right)$.

ROUX Jean-Paul

1966 Faune et flore sacrées dans les sociétés altaïques. Paris: Adrien-Maisonneuve.

SAMPILDENDEV Kh. \& CERENSODNOM D.

1984 Mongol Domog (Légendes mongoles). Oulan Bator: Académie des Sciences, Section de littérature.

SCHAEFFNER André

1968 Origine des instruments de musique. Introduction ethnologique à l'histoire de la musique instrumentale. Paris/La Haye: Mouton.

SCHNEIDER Marius

1960 «Le rôle de la musique dans la mythologie et les rites des civilisations non européennes». In: Histoire de la musique, vol. 1 (publiée sous la direction de Roland Manuel). Paris: Encyclopédie de la Pléiade, p. 131-214.

SMIRNOV B.

1969 «O melose Mongol'skoj Narodnoj Pesni, v Muzyka Narodov Azij i Afriki; kniga 1; izdat». Sovietskij Kompozitor (Moskva): 236-49. 